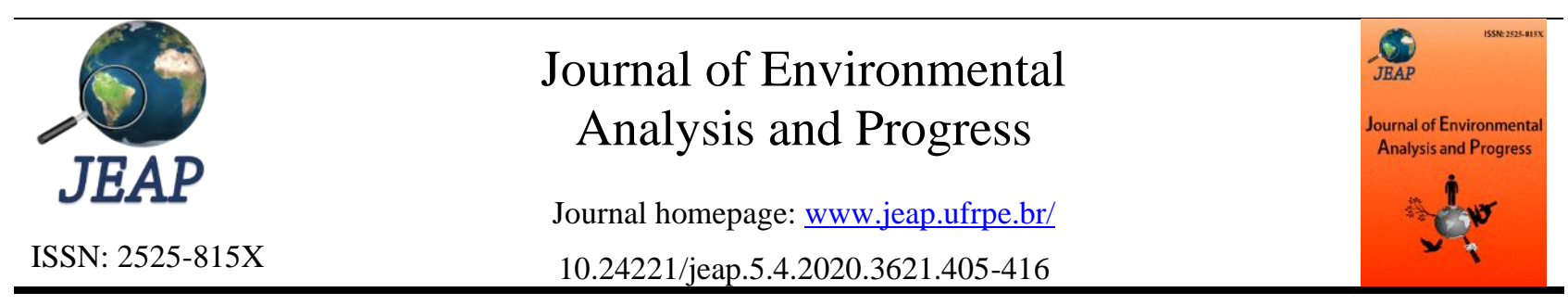

\title{
Caracterização de fertilidade de solo através do uso de colunas de lixiviação alimentadas com efluente tratado
}

\section{Soil’s fertility characterization by using treated effluent leaching columns}

\author{
Marcos Henrique Gomes Ribeiro ${ }^{\mathrm{a}}$, Alanna Maria do Nascimento Bezerra ${ }^{\mathrm{b}}$, Artur Paiva Coutinho ${ }^{\mathrm{b}}$, \\ Elizabeth Amaral Pastich Gonçalves ${ }^{\text {b* }}$
}

${ }^{a}$ Universidade Federal de Pernambuco-UFPE, Centro de Tecnologia e Geociências, Departamento de Engenharia Civil e Ambiental. Av. da Arquitetura s/n, Cidade Universitária, Recife, Pernambuco, Brasil. CEP: 50740-550. E-mail: mhgribeiro@gmail.com.

${ }^{\mathrm{b}}$ UFPE, Centro Acadêmico do Agreste , Núcleo de Tecnologia. Av. Marielle Franco, s/n, km 59, Nova Caruaru, Caruaru, Pernambuco, Brasil. CEP: 55014-900. E-mail: alannamnb@gmail.com, arthur.coutinho@yahoo.com.br, bethpastich@yahoo.com (*Autor correspondente).

\begin{tabular}{l}
\hline A R T I C L E I N F O \\
\hline Recebido 12 Jun 2020 \\
Aceito 18 Nov 2020 \\
Publicado 10 Dez 2020
\end{tabular}

\begin{abstract}
A B S T R A C T
World population growth has increased pressure on natural resources and, in particular, pressure on water resources. The need to provide sufficient water and adequate quality to meet the diverse demands of society is a critical issue in the discussion on sustainable management of water resources. Irrigation is the sector that most contributes to the increase in the demand for water use, considering the need to increase agricultural production to supply the population increase with food and inputs. The treated sewage constitutes a viable attempt to substitute drinking water for less noble uses and that admit smaller restrictive standard. Thus, several initiatives have used sewage treated for agricultural purposes without any maleficent the population and still have the advantage of increasing the soil's element content. The objective of this study was to analyze the improvement of degraded soil attributes from the application of effluent from the stabilization pond with the use of soil columns. The experiment consisted of a control column, fed with distilled water, and two columns fed with the effluent. The physical-chemical characteristics of the leachate and the effluent applied after each application were evaluated, as well as the soil of each column at the end of the experiment. The results obtained were favorable since it was observed a high capacity of color removal, turbidity, and a decrease in the concentration of total coliforms and term tolerant in the leachate from the columns. Concerning soil fertility, an increase in nutrient concentration was observed, especially in phosphorous and nitrogen concentrations. On the other hand, using treated effluent increased sodification potential of the soil.
\end{abstract}

Keywords: Treated effluent, reuse, agriculture, soil columns.

\section{R E S U M O}

O crescimento populacional tem aumentado a pressão sobre os recursos naturais e, em especial, os recursos hídricos. A necessidade de prover água em quantidade suficiente e qualidade adequada para suprir as diversas demandas da sociedade, constitui questão primordial na discussão sobre a gestão sustentável desses recursos. A irrigação é o setor que mais contribui para o aumento da demanda do uso de água, tendo em vista a necessidade de aumento na produção agrícola de forma a suprir o aumento da população com alimentos e insumos. $\mathrm{O}$ esgoto tratado constitui uma tentativa viável de substituição da água potável para usos menos nobres e que admitam menor padrão restritivo, assim, várias iniciativas têm utilizado esgoto tratado para fins agrícolas sem que haja qualquer tipo de maleficio à população e ainda tendo como vantagem o aumento da quantidade de elementos disponíveis no solo. Este estudo objetivou analisar a melhoria dos atributos de solo a partir da 
aplicação de efluente proveniente de lagoa de estabilização com o uso de colunas de solo. O experimento consistiu de uma coluna controle, alimentada com água destilada, e duas colunas alimentadas com o efluente. Foram avaliadas as características físico-químicas dos lixiviados e do efluente aplicado após cada aplicação, e do solo de cada coluna ao final do experimento. Os resultados obtidos se mostraram favoráveis já que foi observada grande capacidade de remoção de cor, turbidez e diminuição na concentração de coliformes totais e termo tolerantes no lixiviado proveniente das colunas. Em relação a fertilidade do solo, foi observado aumento na concentração de nutrientes, em especial nas concentrações de fósforo e nitrogênio. Por outro lado, o uso do efluente tratado também acarretou no aumento do potencial de sodificação do solo.

Palavras-Chave: Efluente tratado, reuso, agricultura, colunas de solo

\section{Introdução}

A destinação adequada de efluentes é parte fundamental no planejamento e gestão dos recursos hídricos, pois minimiza o potencial de poluição e aumenta a possibilidade de reuso de efluentes para fins menos nobres e mais tolerantes (Telles \& Costa, 2007). De acordo com Semura et al. (2007), o reuso se constitui no aproveitamento de águas previamente utilizadas para suprir as necessidades de outros usos, como a irrigação agrícola ou fins paisagísticos. A fertirrigação de culturas alimentícias com efluente, apesar de uma técnica antiga, tem sido cada vez mais estudada como alternativa ao uso convencional de água potável, como observado nos estudos de Almuktar et al. (2015), Almuktar \& Scholz (2016) e Urbano et al. (2017).

Como forma de avaliar, experimentalmente, a viabilidade da aplicação de efluentes tratados no solo, seja para recuperação de área degradada, irrigação de culturas ou monitoramento do deslocamento de elementos pelo perfil do solo, o uso de colunas de solo com amostras da área-alvo, é uma prática bastante utilizada em pesquisas científicas (Lekfeldt, Kjaergaard \& Magid, 2017; Marofi et al., 2015; Stutter, 2015). Segundo Silva (2013), o uso das colunas de solo constitui uma técnica viável e útil, propiciando monitorar o deslocamento de substâncias no perfil do solo. Com a aplicação do efluente líquido, o solo ganha nutrientes, podendo complementar a parcela de matéria orgânica e nutrientes necessários às plantas e ao próprio solo. Por outro lado, Angelakis \& Gikas (2014) ressaltam que altas concentrações de sais em águas de reuso podem reduzir a produtividade agrícola e deteriorar a qualidade do solo para cultivo.

No que concerne ao tratamento de efluentes, o Brasil apresenta condições climáticas bastante favoráveis aos tratamentos biológicos, que apresentam menor custo e são mais sustentáveis, do ponto de vista ambiental. De acordo com a Organização Mundial de Saúde (WHO, 1989), entre os tratamentos biológicos, as lagoas de estabilização são mais apropriadas para o tratamento de efluentes destinados ao reuso agrícola, pois possuem elevada eficiência de remoção de matéria orgânica e de patógenos. Segundo Florêncio et al. (2006), existe a necessidade, em termos gerais, de uma elevada remoção de patógenos em qualquer modalidade de reuso da água. De acordo com Cohim (2006), o uso de efluentes tratados para a irrigação é recomendado nos casos em que não existe contato direto das águas residuárias com as plantas, e sim com as raízes que absorvem os nutrientes.

Neste sentido, o estudo objetivou avaliar a mudança nos atributos químicos e físicos do solo em área da bacia do rio Ipojuca, em Caruaru Pernambuco.

\section{Material e Métodos}

Coleta e caracterização física do solo usado no experimento

O solo usado no estudo foi coletado em área localizada no Campus Acadêmico do Agreste que constitui parte da Universidade Federal Pernambuco, situado no Município de Caruaru. Caruaru é um município pernambucano pertencente à Mesorregião do Agreste, inserido na Microrregião do Vale do Ipojuca. Dista cerca de $130 \mathrm{~km}$ da capital Recife, possui temperatura média anual de $22,7^{\circ} \mathrm{C}$ e vegetação predominante do tipo caatinga (IBGE, 2015). O Clima prevalecente é seco de estepe local, com pouca pluviosidade ao longo do ano, aproximadamente $551 \mathrm{~mm}$ (APAC, 2019). A região, em que está inserido o município de Caruaru, apresenta déficit hídrico e enfrenta diversos problemas relacionados à poluição das águas, sobretudo pela contribuição de efluentes têxteis.

Inicialmente, com o auxílio de trado manual, foi realizada limpeza no local de coleta. Em seguida foi realizada uma (1) coleta com a extração de duas (2) amostras deformadas de solo da camada de 0 a $20 \mathrm{~cm}$ de profundidade. Cada amostra de solo coletada apresentou peso de aproximadamente $400 \mathrm{~g}$. As amostras coletadas foram caracterizadas fisicamente segundo metodologias preconizadas na Embrapa (1997) 
para obtenção de índices físicos para sua classificação. A Tabela 1 apresenta a caracterização física do solo segundo a média obtida para as duas amostras coletadas. De acordo com os resultados obtidos foi possível classificar o solo como areia média a grossa, e, segundo Caputo (1994), o solo foi classificado como seco, pois apresentou umidade inferior a $25 \%$. Ao final da caraterização física, as amostras coletadas foram misturadas e homogeneizadas para a montagem das colunas de lixiviação.

Tabela 1. Caracterização física do solo bruto usado na montagem das colunas de lixiviação. Fonte: Ribeiro, M. H. G. (2020).

\begin{tabular}{lc}
\hline Índice físico & Média \\
\hline $\begin{array}{l}\text { Peso específico dos sólidos } \\
\left(\mathrm{g} \cdot \mathrm{cm}^{-3}\right)\end{array}$ & 1,47 \\
Peso específico natural $\left(\mathrm{g} \cdot \mathrm{cm}^{-3}\right)$ & 1,35 \\
Peso específico seco $\left(\mathrm{g} \cdot \mathrm{cm}^{-3}\right)$ & 1,09 \\
\hline
\end{tabular}

\begin{tabular}{lc}
\hline Porosidade $(\%)$ & 26,17 \\
Indice de vazios & 0,35 \\
Umidade $(\%)$ & 24,15 \\
\hline
\end{tabular}

A caracterização inicial de fertilidade do solo usado no experimento foi realizada pelo Instituto Agronômico de Pernambuco (IPA) segundo metodologias preconizadas por Silva (2009). A estimativa das concentrações de nitrato e amônio do solo foi realizada segundo Silva et al. (2010). O cálculo do percentual de sódio trocável (PST) foi realizado segundo a Equação 1, indicada em Ayers \& Westcott (1994). A Tabela 2 apresenta a caracterização de fertilidade do solo bruto usado na montagem das colunas de lixiviação, obtida considerando os parâmetros analisados.

$$
P S T=\frac{N a}{C a+M g+K+N a+H+A l} \times 100
$$

Tabela 2. Caracterização de fertilidade do solo bruto usado na montagem das colunas de lixiviação. Fonte: Ribeiro, M. H. G. (2020).

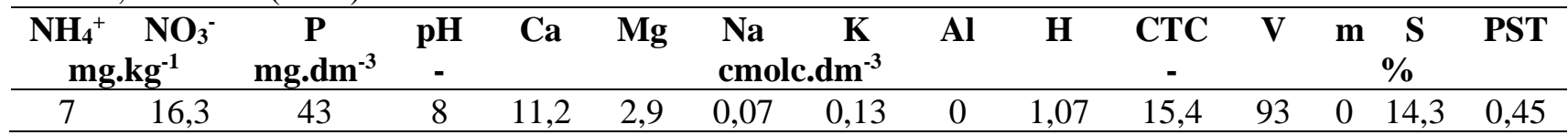

$\mathrm{N}-\mathrm{NH}_{4}{ }^{+}=$nitrogênio na forma de íon amônio; $\mathrm{N}-\mathrm{NO}_{3}{ }^{-}=$nitrogênio na forma de íon nitrato; $\mathrm{P}=$ fósforo total, $\mathrm{pH}=$ potencial hidrogeniônico; $\mathrm{Ca}=$ cálcio; $\mathrm{Mg}=$ magnésio; $\mathrm{Na}=$ sódio; $\mathrm{K}=$ potássio; $\mathrm{Al}=$ alumínio; $\mathrm{H}=$ hidrogênio; $\mathrm{CTC}=$ capacidade de troca catiônica; $\mathrm{V}=$ saturação por bases; $\mathrm{m}=$ saturação por alumínio, $\mathrm{S}=$ soma de bases, PST = percentual de sódio traçável.

Coleta e caracterização físico-química do efluente usado no experimento e dos lixiviados das colunas de solo

O efluente tratado, aplicado nas colunas de solo para lixiviação, foi proveniente de lagoa de polimento da Estação de Tratamento de Efluente (ETE) Rendeiras de Caruaru (-8,283667; 35,936625). A ETE Rendeiras é responsável por tratar $40 \%$ do esgoto da cidade, tendo o rio Ipojuca como corpo receptor (SNIRH, 2019). No período do experimento, operava com a seguinte linha de tratamento: desarenadores do tipo canal retangular; tratamento anaeróbio por meio de reatores tipo UASB (reator anaeróbio de fluxo ascendente e manta de lodo); tratamento aeróbio por meio de lagoa aerada com difusores de membrana; lagoa de sedimentação e lagoa de polimento final (INTERÁGUAS, 2017).

Foi realizada uma (1) coleta de efluente proveniente de lagoa de maturação com recipiente de $5 \mathrm{~L}$ A caraterização físico-química do efluente e dos lixiviados das colunas de solo foi realizada segundo as metodologias preconizadas em APHA (2012). Para os parâmetros $\mathrm{pH}$, oxigênio dissolvido (OD), temperatura, salinidade, condutividade elétrica (CE), cor e turbidez, foi realizado o monitoramento diário para o efluente e lixiviados ao longo do ciclo experimental. Para os parâmetros demanda química de oxigênio (DQO), coliformes totais e termotolerantes, fósforo e alcalinidade total, realizou-se a caracterização do lixiviado para o total acumulado referente a cada coluna de solo ao final do experimento. A Tabela 3 apresenta a caracterização físico-química do efluente tratado usado nas colunas de solo. Durante todo o período de experimento (sete dias), o efluente permaneceu acondicionado sob refrigeração.

Tabela 3. Caracterização do efluente tratado usado como fonte de alimentação nas colunas de lixiviação. Fonte: Ribeiro, M. H. G. (2020).

\begin{tabular}{lc}
\hline Variável & Resultado \\
\hline $\mathrm{pH}$ & 8,24 \\
Oxigênio dissolvido $\left(\mathrm{mg} . \mathrm{L}^{-1}\right)$ & 4,92 \\
Temperatura $\left({ }^{\circ} \mathrm{C}\right)$ & 18,80 \\
Salinidade $(\mathrm{ppm})$ & 0,46 \\
Condutividade elétrica $\left(\mu \mathrm{S} . \mathrm{cm}^{-1}\right)$ & $1.196,76$ \\
Cor $(\mathrm{uH})$ & 329,71 \\
Turbidez $(\mathrm{UNT})$ & 65,77 \\
Demanda química de oxigênio & 229,48 \\
$\left(\mathrm{mg} . \mathrm{L}^{-1}\right)$ & $8,66 \times 10^{4}$ \\
Coliformes totais $\left(\mathrm{NMP} .100 \mathrm{~mL}^{-1}\right)$ & \\
\hline
\end{tabular}




\begin{tabular}{lc}
\hline Coliformes termotolerantes & $3,00 \times 10^{2}$ \\
(NMP.100 mL & \\
Fósforo $\left(\mathrm{mg} \cdot \mathrm{L}^{-1}\right)$ & 13,40 \\
Alcalinidade total $\left(\mathrm{mg} \cdot \mathrm{L}^{-1}\right)$ & 80,00 \\
\hline
\end{tabular}

A água utilizada ao longo do experimento foi do tipo destilada. Dessa forma, por não apresentar valores consideráveis em relação aos parâmetros avaliados para o efluente e os lixiviados, os mesmos tiveram seus dados de caracterização omitidos.

\section{Montagem das colunas de solo}

Para a montagem das colunas de solo foram utilizadas colunas em acrílico com $6 \mathrm{~cm}$ de diâmetro interno, $7 \mathrm{~cm}$ de diâmetro externo e $30 \mathrm{~cm}$ de altura. As colunas foram constituídas de material transparente (acrílico) e não receberam nenhum tipo de cobertura ao longo do experimento, ou seja, permaneceram expostas à luz. Antes do preenchimento das colunas, o solo foi seco ao ar livre e destorroado. O preenchimento das colunas foi realizado de modo a reproduzir as condições do solo natural. Cada coluna foi preenchida com $763,41 \mathrm{~g}$, referente à $565,49 \mathrm{~cm}^{3}$ de solo para a obtenção de peso específico de $1,35 \mathrm{~g}$, equivalente ao peso específico natural do solo, obtido por meio dos ensaios iniciais de caracterização física. A Figura 1 apresenta o aparato experimental das colunas de lixiviação.
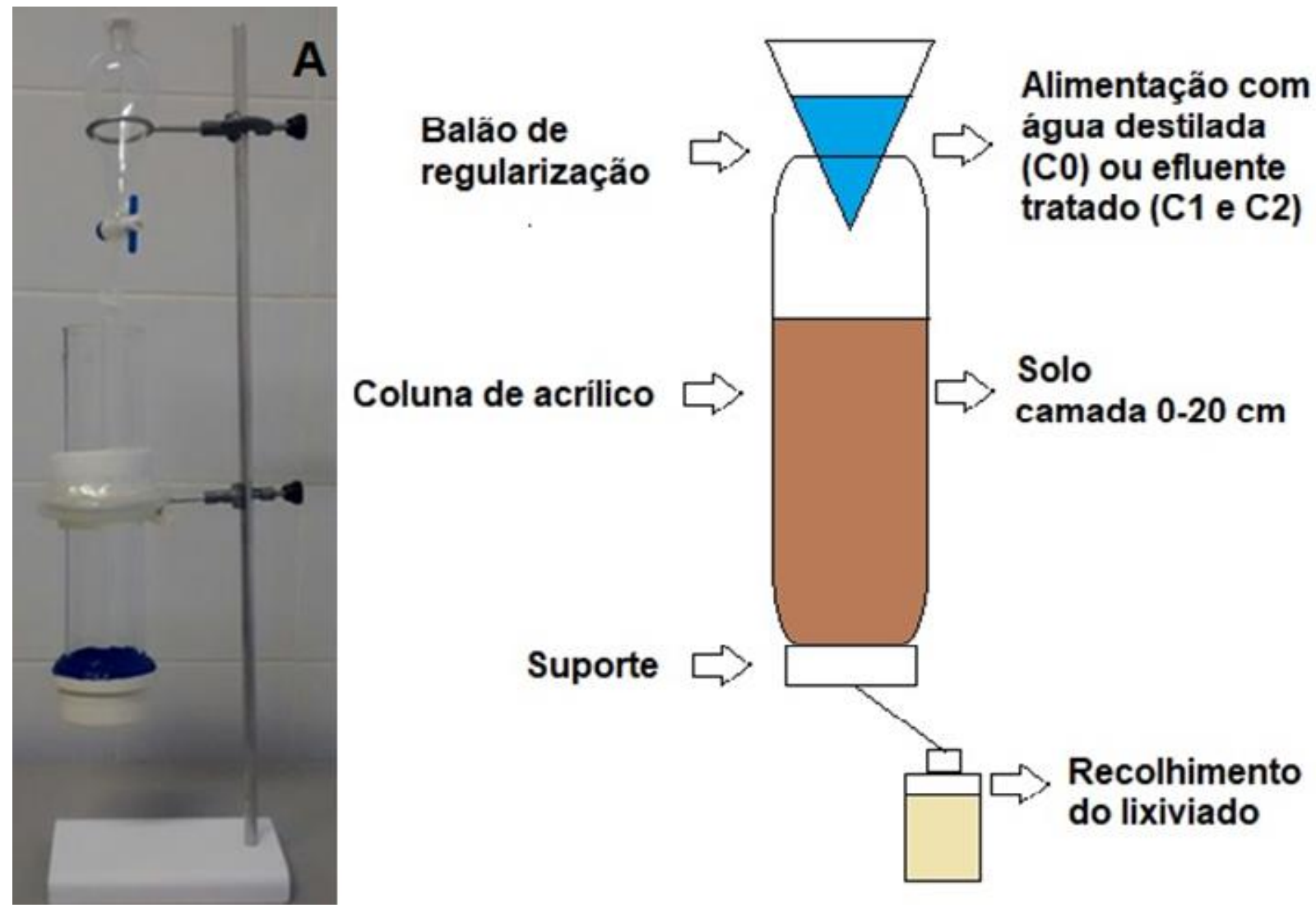

Figura 1. Representação do aparato utilizado nas colunas de lixiviação usadas no experimento. A) Modelo de suporte para coluna e balão de regularização. B) Desenho esquemático demonstrando os constituintes do experimento. Fonte: Ribeiro, M. H. G. (2020).

Os solos acondicionados nas colunas foram saturados com água destilada $(500 \mathrm{~mL})$ em fluxo ascendente por quatro dias, com o objetivo de eliminação de bolhas de ar e homogeneização da umidade (Matos, Gariglio \& Monaco, 2013). Após a infiltração completa da água nas colunas e início do escoamento permanente, a aplicação de água foi interrompida e deu-se início a alimentação. Com o objetivo de não formar lâmina superficial e garantir faixa homogênea de alimentação, foi estabelecida uma vazão de 9,09 mL.min ${ }^{-1}$ para as colunas, mantida através de funil de regularização. Cada coluna recebeu alimentação diária, de forma manual em batelada, com $100 \mathrm{~mL}$ de efluente $(\mathrm{C} 1$ e $\mathrm{C} 2$ ) ou água destilada $(\mathrm{C} 0)$ durante o tempo total de experimento ( 7 dias). Ao final do experimento, cada coluna recebeu $700 \mathrm{~mL}$ de alimentação (efluente ou água destilada).

Foram montadas ao todo, três colunas de lixiviação, sendo uma coluna-controle, alimentada com água destilada (C0) e duas colunas alimentadas com efluente tratado, $\mathrm{C} 1$ e $\mathrm{C} 2$ (réplicas). Cada coluna foi conectada a um béquer, onde, após a passagem do fluido (água destilada ou 
efluente tratado) pelo solo, o lixiviado foi recolhido para posterior caracterização físico-química. A Tabela 4 especifica o delineamento adotado no experimento.

Tabela 4. Delineamento experimental adotado. Fonte: Ribeiro, M. H. G. (2020).

\begin{tabular}{|c|c|c|}
\hline Coluna & Alimentação & Lixiviado \\
\hline $\begin{array}{l}\mathrm{C} 0 \\
\text { (controle) }\end{array}$ & $\begin{array}{c}\text { Água } \\
\text { destilada }\end{array}$ & Lixiviado-água \\
\hline $\mathrm{C} 1$ & $\begin{array}{c}\text { Efluente } \\
\text { tratado }\end{array}$ & $\begin{array}{l}\text { Lixiviado- } \\
\text { efluente-1 }\end{array}$ \\
\hline $\mathrm{C} 2$ & $\begin{array}{c}\text { Efluente } \\
\text { tratado }\end{array}$ & $\begin{array}{l}\text { Lixiviado- } \\
\text { efluente-2 }\end{array}$ \\
\hline
\end{tabular}

\section{Resultados e Discussão}

Ao passar pelas colunas de solo, o efluente apresentou redução média nos valores de turbidez e cor (Figura 2). Os parâmetros obtiveram redução, respectivamente, de $89,6 \%$ (Figura 2A) e $89,5 \%$ (Figura 2B). Em lagoas de estabilização, como a lagoa de polimento, a turbidez é resultado, principalmente, das elevadas densidades de microalgas suspensas. Neste caso, o parâmetro pode ser utilizado como indicador da remoção da demanda química de oxigênio, sólidos em suspensão e sólidos voláteis (Nguyen et al., 2014). Dessa forma, a redução nos valores de turbidez indicou o provável depósito de sólidos no solo das colunas de lixiviação.
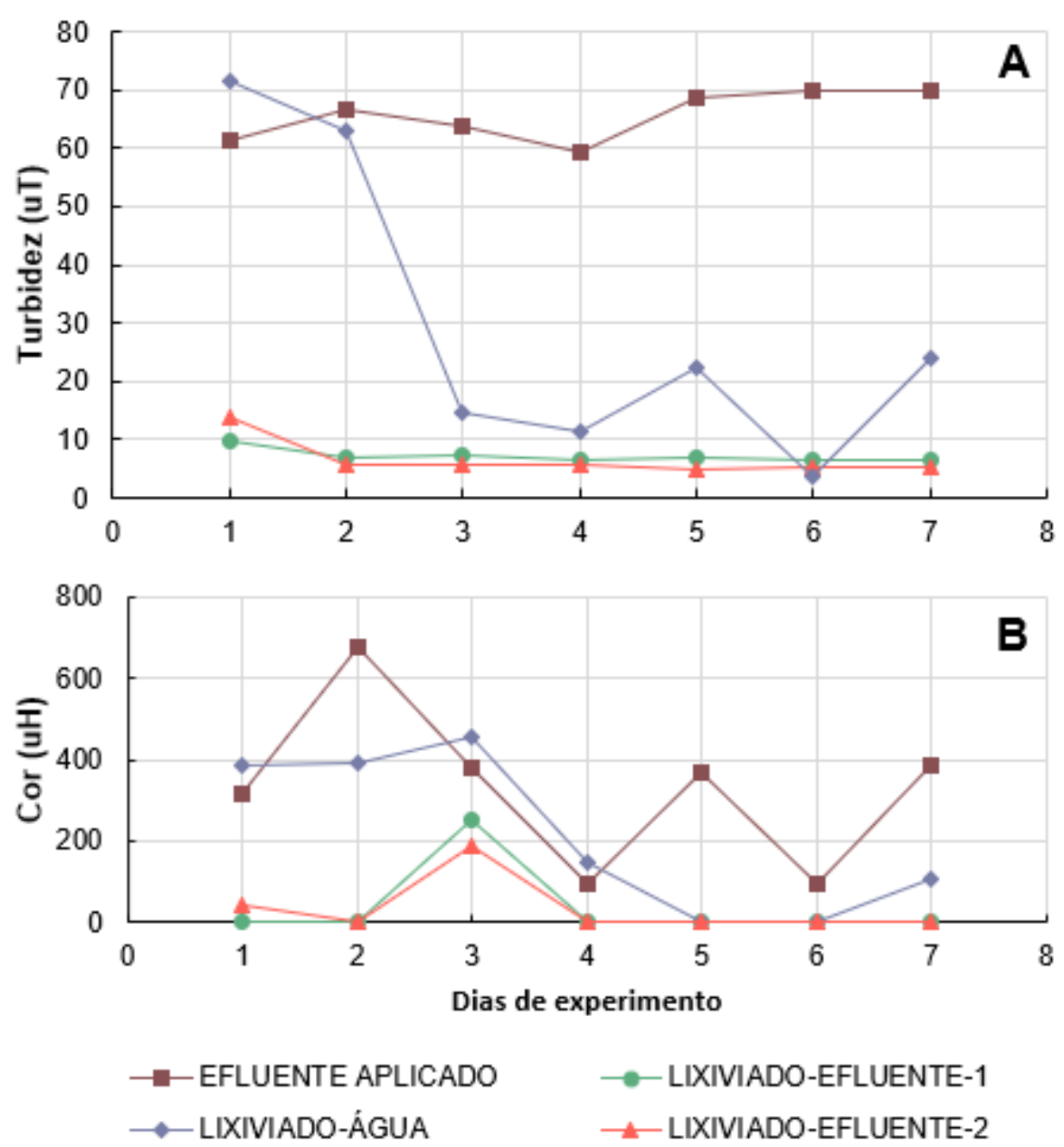

Figura 2. Comportamento dos parâmetros de turbidez e cor no efluente aplicado como fonte de alimentação nas colunas 1 e 2 e nos lixiviados das colunas 0,1 e 2, ao longo do experimento: A. turbidez; B. cor. Fonte: Ribeiro, M. H. G. (2020).

A cor também está associada à presença de sólidos dissolvidos, principalmente material em estado coloidal orgânico e inorgânico (Metcalf \& Eddy, 1991). Dessa forma, a diminuição da cor apresentada nos resultados dos lixiviados das colunas alimentadas com efluente tratado indicaram que a passagem do efluente pelo solo poderia ter contribuído para o aumento do teor de material orgânico do mesmo. A matéria orgânica exerce importante efeito benéfico sobre as propriedades do solo, de forma física, química e biológica, contribuindo, substancialmente, para o crescimento e o desenvolvimento das plantas com 
suprimento constante de nitrogênio orgânico (Kiehl, 1985).

A condutividade elétrica no efluente aplicado permaneceu em torno de $1.200 \mu \mathrm{S} . \mathrm{cm}^{-1}$ (Figura 3). Segundo Almeida (2010), o intervalo usual da CE nas águas de irrigação varia de 0 a $3.000 \mu \mathrm{S} . \mathrm{cm}^{-1}$. De acordo com Cordeiro (2001), as águas de irrigação podem ser classificadas conforme a Tabela 5, sendo águas com salinidade muito alta indicadas apenas para uso em solos permeáveis e em plantas que apresentem alta tolerância aos sais. O efluente do presente estudo esteve classificado como apresentando alta salinidade.

Tabela 5. Classificação das águas de irrigação segundo o valor de condutividade elétrica. Fonte: Adaptado de Cordeiro (2001).

\begin{tabular}{lcc}
\hline Classe & Salinidade & CE $\left(\boldsymbol{\mu S} . \mathbf{c m}^{-\mathbf{1}}\right)$ \\
\hline C1 & Baixa & $<250$ \\
C2 & Média & $250-750$ \\
C3 & Alta & $700-2.250$ \\
C4 & Muito alta & $>2.250$ \\
\hline
\end{tabular}

Diversos autores discutiram valores maiores de condutividade elétrica em efluentes como Silva Júnior et al. (2019) com $3.000 \mu$ S.cm $^{-}$ ${ }^{1} \mathrm{e}$ Sandri \& Rosa (2017) com $2.010 \mu \mathrm{S} . \mathrm{cm}^{-1}$.
Urbano et al. (2017), obtiveram com valores menores que o do presente estudo, $830 \mu \mathrm{S} . \mathrm{cm}^{-1}$.

Os resultados de condutividade elétrica mostraram que a partir de determinada leitura, houve convergência das curvas de condutividade do efluente bruto e o do lixiviado das colunas $\mathrm{C} 1 \mathrm{e}$ $\mathrm{C} 2$. Este fato de deveu à saturação do solo em relação à quantidade de íons que, obtidos a partir do efluente, se somaram aos íons provenientes do próprio solo. Sendo, dessa forma, a partir de certo momento, totalmente lixiviados com o soluto à medida que se atingiu o ponto de equilíbrio. De acordo com Jeong, Kim \& Jang (2016), o limite máximo de valor de condutividade atingido pelo efluente no presente estudo $\left(1.200 \mu \mathrm{S} . \mathrm{cm}^{-1}\right)$, está dentro dos valores aconselhados para o uso com águas de irrigação segundo legislações internacionais, como a italiana e a israelense.

Foi possível perceber que, com a saturação do solo em relação ao efluente, é possível uma futura salinização da camada (Kiziloglu et al., 2008). No entanto, em um estudo publicado por Rusan, Hinnawi \& Rousan (2007), os sais se acumularam em maiores proporções nas camadas mais profundas. Isto deve estar atrelado ao manejo de uma irrigação que garanta sua lixiviação abaixo nível de profundidade dos sistemas radiculares das plantas, a fim de que não afete negativamente a atividade microbiana no solo, o crescimento das plantas e a produtividade do solo.
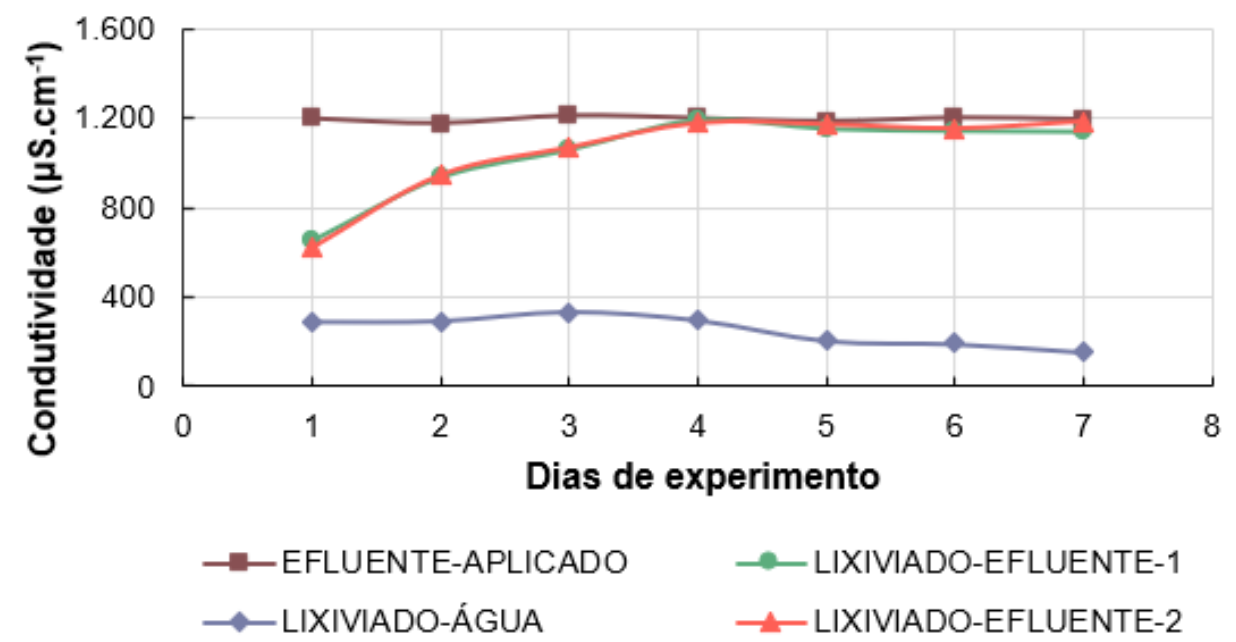

Figura 3. Comportamento do parâmetro condutividade elétrica no efluente aplicado como fonte de alimentação nas colunas 1 e 2 e nos lixiviados das colunas 0,1 e 2, ao longo do experimento. Fonte: Ribeiro, M. H. G. (2020).

A temperatura (Figura 4A) não apresentou diferenças expressivas. O efluente, ao ser aplicado às colunas, apresentou temperatura mais baixa que às apresentadas no lixiviado, visto o mesmo permaneceu acondicionado em geladeira durante $\mathrm{o}$ período experimental.
O oxigênio dissolvido do efluente apresentou maior valor comparado aos observados nos lixiviados (Figura 4B). O efluente proveniente da lagoa de maturação é rico em algas fotossintetizantes que produzem OD, no entanto, após a lixiviação o OD é perdido e não mais reposto pelo processo de fotossíntese acarretando 
diminuição da sua concentração no lixiviado como esperado.

Em relação ao $\mathrm{pH}$ (Figura 4C), os valores estiveram próximos à neutralidade, favorecendo o equilíbrio dos compostos químicos e uma normalidade na taxa de crescimento dos microrganismos. Segundo Jeong, Kim \& Jang (2016), a concentração de hidrogênio, medida a partir do pH, é um parâmetro fundamental para a avaliação do potencial de reuso da água para irrigação, cujo valores ideais encontram-se na faixa de 6,5 a 8,4 . Valores de $\mathrm{pH}$ fora dessa faixa, ainda assim, podem ser usados para fins de irrigação, no entanto, apresentam potencial para causar desequilíbrios nutricionais ou conter íons nocivos ao solo.
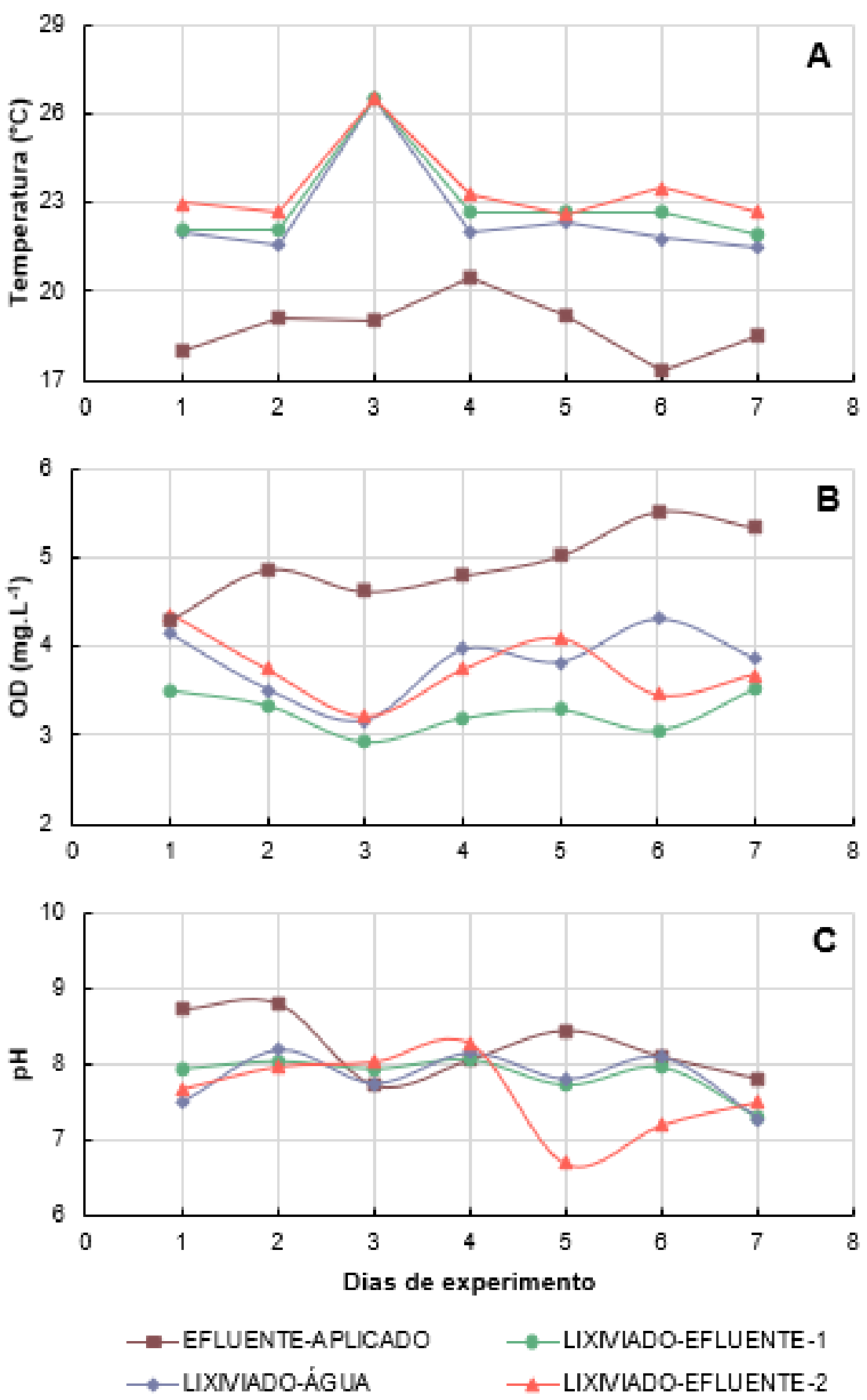

Figura 4. Comportamento dos parâmetros no efluente aplicado como fonte de alimentação nas colunas 1 e 2 e nos lixiviados das colunas 0,1 e 2, ao longo do experimento: A. temperatura; B. oxigênio dissolvido-OD; C. pH. Fonte: Ribeiro, M. H. G. (2020).

A Tabela 6 apresenta os resultados de demanda química de oxigênio (DQO) e fósforo, obtidos no efluente e nos lixividos das colunas de saturação. 
Tabela 6. Valores de demanda química de oxigênio (DQO) e fósforo para efluente aplicado como fonte de alimentação nas colunas 1 e 2 e nos lixiviados das colunas 0,1 e 2, ao longo do experimento. Fonte: Ribeiro, M. H. G. (2020).

\begin{tabular}{lcccc}
\hline Variável & Efluente & \multicolumn{3}{c}{ Lixiviados } \\
& aplicado & Agua destilada $(\mathbf{C 0})$ & Efluente-1 $(\mathbf{C 1})$ & Efluente-2 $(\mathbf{C 2})$ \\
\hline DQO $\left(\mathrm{mg} . \mathrm{L}^{-1}\right)$ & 229,48 & 75,77 & 175,81 & 171,80 \\
Fósforo $\left(\mathrm{mg} . \mathrm{L}^{-1}\right)$ & 13,39 & 1,43 & 0,96 & 0,94 \\
\hline
\end{tabular}

Na Tabela 6, a coluna C0 (controle) alimentada com água destilada, apresentou valor de DQO no lixiviado de $79,1 \mathrm{mg} . \mathrm{L}^{-1}$, que representa parte da matéria orgânica presente inicialmente no solo. As colunas $\mathrm{C} 1$ e $\mathrm{C} 2$, que foram alimentadas com efluente, apresentaram valores de $175,8 \mathrm{e}$ $171,8 \mathrm{mg} . \mathrm{L}^{-1}$, respectivamente. Isto evidencia que houve a incorporação de matéria orgânica proveniente do efluente no solo destas colunas de aproximadamente 23 e $25 \%$, respectivamente. Isto também foi observado por Rusan, Hinnawi \& Rousan (2007).

A matéria orgânica atua beneficamente no solo pois sequestra metais, impede a mobilidade e o risco de lixiviação destes e atua como reservatório de nutrientes, como observado por Gwenzi \& Munondo (2008) e Becerra-Castro et al. (2015). Siebe (1998) realça que a irrigação com águas residuais aumenta o conteúdo de matéria orgânica no solo, o que ajuda a estabilizar a estrutura do solo e tem uma influência positiva na retenção de água e nas trocas gasosas. Arienzo et al. (2009), por sua vez, destacam que a matéria orgânica das águas residuárias pode diminuir o potencial de erosão do solo, aumentar a troca de cátions, tamponar o $\mathrm{pH}$ e promover o crescimento de organismos benéficos no solo.

Na coluna C0 (controle) observou-se uma concentração de fósforo de $1,8 \mathrm{mg} . \mathrm{L}^{-1}$, proveniente da lixiviação deste elemento presente no solo bruto (Tabela 2). O valor obtido de fósforo do lixiviado das colunas $\mathrm{C} 1$ e $\mathrm{C} 2$ foi aproximadamente $93 \%$ menor que o valor observado no efluente bruto, demostrando que parte do fósforo do efluente foi incorporado ao solo.

A Tabela 7 apresenta os resultados para coliformes totais e termotolerantes para o efluente e para os lixiviados das colunas que foram saturadas com o efluente ( $\mathrm{C} 1$ e $\mathrm{C} 2)$.

Tabela 7. Valores de coliforme totais e termotolerantes para efluente aplicado como fonte de alimentação nas colunas 1 e 2 e nos lixiviados das colunas 1 e 2, ao final do experimento. Fonte: Ribeiro, M. H. G. (2020).

\begin{tabular}{lccc}
\hline \multirow{2}{*}{ Fonte hídrica } & Coliformes totais & \multicolumn{2}{c}{ Coliformes termotolerantes } \\
\cline { 2 - 4 } & & $\left(\mathbf{N M P . 1 0 0 . \mathbf { m L } ^ { - 1 }}\right)$ \\
\hline Efluente aplicado & $8,66 \times 10^{4}$ & $3,00 \times 10^{2}$ \\
Lixiviado -efluente-1 & $\geq 2.419,60^{*}$ & $2,80 \times 10$ \\
Lixiviado-efluente-2 & $\geq 2.419,60^{*}$ & $3,36 \times 10$ \\
\hline
\end{tabular}

* = acima do limite de detecção.

Em relação aos coliformes termotolerantes (Tabela 7), houve redução na concentração no efluente para o lixiviado de $90,5 \%$ na coluna $\mathrm{C} 1 \mathrm{e}$ $88,9 \%$ de remoção na coluna C2. Este resultado indicou baixa probabilidade de contaminação fecal no lençol freático e bom indicativo do solo utilizado como filtro de pós tratamento de efluente. Por outro lado, o uso de águas residuais para irrigação de solos pode ser uma fonte de bactérias benéficas, como bactérias envolvidas no ciclo do nitrogênio ou outras com capacidade de remediar contaminantes no solo, como observado por Becerra-Castro et al. (2015).

A Tabela 8 apresenta os resultados relativos à fertilidade do solo ao final do ciclo experimental. A coluna $\mathrm{C} 0$ foi o controle, e aquela que recebeu alimentação com água destilada, as colunas $\mathrm{C} 1$ e $\mathrm{C} 2$ foram as que receberam alimentação com efluente tratado.

Tabela 8. Caracterização de fertilidade do solo usados nas colunas de lixiviação ao final dos sete dias de experimento. Fonte: Ribeiro, M. H. G. (2020).

\begin{tabular}{lcccc}
\hline Variável & Unidade & $\begin{array}{c}\text { Coluna controle } \\
(\mathbf{C 0})\end{array}$ & $\begin{array}{c}\text { Coluna efluente-1 } \\
(\mathbf{C 1})\end{array}$ & $\begin{array}{c}\text { Coluna efluente-2 } \\
(\mathbf{C 2})\end{array}$ \\
\hline $\mathrm{N}-\mathrm{NH}_{4}{ }^{+}$ & $\mathrm{mg} \cdot \mathrm{kg}^{-1}$ & 7,00 & 0,00 & 0,00 \\
$\mathrm{~N}-\mathrm{NO}_{3}{ }^{-}$ & $\mathrm{mg} \cdot \mathrm{dm}^{-3}$ & 37,33 & 35,00 & 23,33 \\
$\mathrm{P}$ & - & 8,30 & 68,00 & 62,00 \\
$\mathrm{pH}$ & & & 8,10 & 7,80 \\
\hline
\end{tabular}




\begin{tabular}{|c|c|c|c|c|}
\hline $\mathrm{Ca}^{+2}$ & \multirow{4}{*}{ cmolc. dm ${ }^{-3}$} & 11,20 & 10,00 & 9,50 \\
\hline $\mathrm{Mg}^{+2}$ & & 3,15 & 2,30 & 2,35 \\
\hline $\mathrm{Na}^{+}$ & & 0,20 & 1,20 & 1,30 \\
\hline $\mathrm{K}^{+}$ & & 0,13 & 0,26 & 0,28 \\
\hline $\mathrm{Al}^{+3}$ & & 0,00 & 0,00 & 0,00 \\
\hline $\mathrm{H}^{+}$ & & 0,16 & 0,06 & 0,41 \\
\hline CTC & - & 14,90 & 13,60 & 13,40 \\
\hline$S$ & \multirow{4}{*}{$\%$} & 14,70 & 13,60 & 13,40 \\
\hline V & & 99,00 & 99,00 & 97,00 \\
\hline $\mathrm{m}$ & & 0,00 & 0,00 & 0,00 \\
\hline PST & & 1,35 & 8,68 & 9,39 \\
\hline
\end{tabular}

$\mathrm{N}-\mathrm{NH}_{4}{ }^{+}=$nitrogênio na forma de íon amônio; $\mathrm{N}-\mathrm{NO}_{3}{ }^{-}=$nitrogênio na forma de íon nitrato; $\mathrm{P}=$ fósforo total, $\mathrm{pH}=$ potencial hidrogeniônico; $\mathrm{Ca}=$ cálcio; $\mathrm{Mg}=$ magnésio; $\mathrm{Na}=$ sódio; $\mathrm{K}=$ potássio; $\mathrm{Al}$ = alumínio; $\mathrm{H}=$ hidrogênio; $\mathrm{CTC}=$ capacidade de troca catiônica; $\mathrm{V}=$ saturação por bases; $\mathrm{m}=$ saturação por alumínio, $\mathrm{S}=$ soma de bases, PST = percentual de sódio traçável.

A concentração de nitrogênio, sob a forma de nitrato, no solo da coluna $\mathrm{C} 0$ (controle) reduziu ao final do experimento (Tabela 5), visto que parte dele lixiviou no solo. Em relação ao nitrogênio sob a forma amoniacal, ele permaneceu constante. A concentração nas colunas C1 e C2 mostrou aumento (Tabela 8), mostrando que parte do nitrato do esgoto foi incorporado no solo sob a forma de nitrato e a parcela referente ao amônio foi provavelmente nitrificada (Gloaguen et al., 2007). Segundo Hadas et al. (1999), as quantidades de nitrato detectadas em solos são derivadas da decomposição de raízes de culturas ou adubo acondicionado na forma de esterco ou lixo urbano compostado. Além disso, apenas uma porcentagem limitada do conteúdo total de nutrientes de uma água residual de esgoto municipal está em forma imediatamente disponível para a incorporação nos solos (Magesan et al., 1998).

Não foram observadas diferenças expressivas para os parâmetros de $\mathrm{pH}$ do solo, cálcio, magnésio e alumínio, ao final do experimento. Em relação ao fósforo, corroborando os resultados encontrados na análise do lixiviado, ocorreu incorporação no solo das colunas $\mathrm{C} 1$ e C2 em torno de $58 \%$ e $44 \%$ respectivamente, em comparação ao valor encontrado na análise do solo bruto. A concentração de fósforo disponível nas amostras de solo irrigadas com efluente foi maior, indicando que a matéria orgânica contida no efluente pode proteger o $\mathrm{P}$ da insolubilização (Bedbabis et al., 2014). Através da alimentação com efluente, foi incorporado ao solo $9,38 \mathrm{mg}$ de fósforo para $\mathrm{C} 1$ e $\mathrm{C} 2$.

Ocorreu aumento nas concentrações de potássio, tornando-se um suprimento importante, visto que, em ambientes de solo tropical, o suprimento de $\mathrm{K}^{+}$é um problema agronômico por esse elemento ser pouco adsorvido, altamente solúvel e facilmente lixiviado (Gloaguen et al., 2007). Arienzo et al. (2009) observaram aumento dos níveis de potássio, com aplicação terrestre de águas residuais, mais rapidamente do que com fertilizantes inorgânicos convencionais.

Houve também aumento do teor de sódio no solo das colunas alimentadas com efluente (Tabela 5), o que está ligado diretamente ao risco de salinização. A alta incorporação de $\mathrm{K}^{+} \mathrm{e} \mathrm{Na}^{+}$no solo também foram observadas por Gwenzi \& Munondo (2008).

$\mathrm{O}$ aumento de nitrato e potássio nas amostras de solos irrigados com efluente deve-se ao fato das águas residuais apresentarem quantidades significativas desses nutrientes que são essenciais para melhorar o crescimento das plantas e os níveis de fertilidade e produtividade do solo, podendo substituir ou reduzir o uso de fertilizantes sintéticos na agricultura (Rusan, Hinnawi \& Rousan, 2007; Becerra-Castro et al., 2015).

A diminuição dos valores de capacidade de troca catiônica (CTC) e da soma de bases trocáveis, que acarretou o aumento do índice de saturação por bases $(\mathrm{V})$, indica a melhoria da fertilidade do solo após a alimentação com o efluente tratado de sódio trocável e a razão de absorção de sódio. Adicionalmente foi observado um aumento dos valores no solo após a aplicação do efluente. Isto é preocupante devido ao risco de salinização e sodização do solo, processo particularmente intensificado em regiões semiáridas e que necessita de avaliação periódica da situação.

Os valores de percentual de sódio trocável (PST) foram muito superiores nos solos irrigados por efluente (Tabela 8), em relação ao valor do solo original usado no experimento (Tabela 2). Enquanto o solo original apresentou valor de PST igual a $0,45 \%$, os solos das colunas $\mathrm{C} 1$ e $\mathrm{C} 2$, alimentadas com efluente tratado, apresentaram valores de 8,68 e 9,39\%, respectivamente. Segundo a classificação proposta por Massoud (1974), apresentada na Tabela 9, o solo passou de não- 
sódico para ligeiramente sódico. Isto indica que houve aumento da preponderância do íon $\mathrm{Na}^{+} \mathrm{em}$ reação aos demais.

Tabela 9. Classificação do solo segundo o valor de PST. Fonte: Adaptado de Massoud (1974).

\begin{tabular}{lc}
\hline Solo & PST $(\boldsymbol{\%})$ \\
\hline Não-sódico & $<7$ \\
Ligeiramente sódico & $7-10$ \\
Mediamente sódico & $11-20$ \\
Fortemente sódico & $21-30$ \\
Excessivamente sódico & $>30$ \\
\hline
\end{tabular}

O aumento dos valores de PST nas amostras de solo irrigados com efluente deveu-se provavelmente a acumulações de $\mathrm{Na}^{+}$e a uma taxa reduzida de infiltração de água que afetou negativamente uma lixiviação mais acentuada de $\mathrm{Na}^{+}$(Bedbabis et al., 2014). Corroborando com o presente estudo Gloagen et al. (2007) também observaram impacto nos valores de $\mathrm{Na}^{+}$no solo através do aumento do sódio pela irrigação. Porém, Fonseca et al. (2007) observaram que a irrigação por efluente tratado forneceu nutrientes minerais disponíveis para o solo, podendo diminuir o efeito deletério do $\mathrm{Na}^{+}$nas plantas.

\section{Conclusão}

A remoção de cor, turbidez e coliformes totais termotolerantes indicou o efeito benéfico do solo como filtro para a remoção de matéria orgânica, materiais particulados e dissolvidos e patógenos, acarretando maior segurança ambiental em relação à contaminação de aquíferos. A redução observada nos valores de DQO no lixiviado das colunas alimentadas com efluente tratado indicou possível incorporação da matéria orgânica presente no efluente, reafirmando o potencial de filtração do solo e a diminuição de possível contaminação ambiental pelo lançamento do efluente no emissário.

Para os parâmetros de fertilidade do solo, houve aumento dos teores da maioria dos elementos, em especial nos valores de fósforo e nitrogênio. Este fato evidencia o benefício do reuso de efluentes na recuperação e aumento de fertilidade em solos que sofreram perda de elementos essenciais para o desenvolvimento de plantas. Os valores obtidos para PST indicaram o aumento do potencial de sodificação do solo pelo uso efluente tratado.

É necessária uma escolha adequada do tipo de efluente utilizado, visando não causar danos à estrutura física ou química do solo e que atenda tanto as necessidades desejadas quanto ao o tipo de uso.

\section{Agradecimentos}

Os autores agradecem ao Laboratório de Engenharia Ambiental, parte do Centro Acadêmico do Agreste da Universidade Federal de Pernambuco, pelo suporte para a realização deste estudo.

\section{Referências}

Almeida, O. A. 2010. Qualidade da água de irrigação. Embrapa, Cruz das Almas, Bahia.

Almuktar, S. A. A. A. N.; Scholz, M. 2016. Mineral and biological contamination of soil and Capsicum annuum irrigated with recycled domestic wastewater. Agricultural Water Management, 167, 95-109. doi: 10.1016/j.agwat.2016.01.008

Almuktar, S. A. A. A. N.; Scholz, M.; Al-Isawi, R. H. K.; Sani, A. 2015. Recycling of domestic wastewater treated by vertical-flow wetlands for irrigating chillies and sweet peppers. Agricultural Water Management, 149, 1-22. doi: 10.1016/j.agwat.2014.10.025

Angelakis, A. N.; Gikas, P. 2014. Water reuse: overview of current practices and trends in the world with emphasis on EU states. Water Utility Journal, 8, 67-78.

APAC - Agência Pernambucana de Águas e Clima. Monitoramento das chuvas no estado de Pernambuco. 2019. Disponível em: http://www.apac.pe.gov.br/meteorologia/chu vas-rmr.php. Acesso em: 09 set. 2019.

Arienzo, M.; Christen, E. W.; Quayle, W.; Kumar, A. 2009. A review of the fate of potassium in the soil-plant system after land application of wastewater. Journal of Hazardous Materials, 164, 415-422. doi: 10.1016/j.jhazmat.2008.08.095

Ayers, R. S.; Westcott, D. W. 1994. Water quality for agriculture. FAO, Rome, Italy.

Baird, R. B.; Eaton, A. D.; Rice, E. W. (ed.) Standard Methods for the Examination of Water and Wastewater. 22. ed. Washington, D.C., USA: American Public Health Association (APHA), American Water Works Association (AWWA) and Water Environment Federation (WEF). 2012.

Becerra-Castro, C.; Lopes, A. R.; Vaz-Moreira, I.; Silva, E. F.; Manaia, C. M.; Nunes, O. C. 2015. Wastewater reuse in irrigation: A microbiological perspective on implications in soil fertility and human and environmental health. Environmental International, 75, 117135. doi: 10.1016/j.envint.2014.11.001

Bedbabis, S.; Rouina, B. B.; Boukhris, M.; Ferrara, G. 2014. Effect of irrigation with treated wastewater on soil chemical properties and infiltration rate. Journal of Environmental 
Management, 133, 45-50. doi: 10.1016/j.jenvman.2013.11.007

Caputo, H. P. 1994. Mecânica dos Solos e suas Aplicações, Livro Técnico, Vol. 1, Rio de Janeiro, Brasil.

Cohim, E. 2006. Polimento de efluente de UASB com filtros intermitentes de areia. Dissertação de Mestrado, Universidade Federal da Bahia. Salvador, Bahia, Brasil. 128p.

Cordeiro, G. G. 2001. Qualidade de água para fins de irrigação: conceitos básicos e práticos. Petrolina - PE: Embrapa Semiárido.

Claessen, M. E. C. (org.). 1997. Manual de métodos de análise de solo. 2. ed. rev. atual. Centro Nacional de Pesquisa de Solo. Rio de Janeiro: EMBRAPA-CNS. Disponível em: https://www.agencia.cnptia.embrapa.br/Repo sitorio/Manual+de+Metodos_000fzvhotqk02 wx5ok0q43a0ram31wtr.pdf. Acesso em: 29 maio 2018.

Florencio, L.; Bastos, R. K. X.; Aisse, M. M. (coord). 2006. Tratamento e utilização de esgotos sanitários. ABES, FINEP/PROSAB. Rio de Janeiro.

Fonseca, A. F.; Melfi, A. J.; Monteiro, F. A.; Montes, C. R.; Almeida, V. V.; Herpin, U. 2007. Treated sewage effluent as a source of water and nitrogen for Tifton 85 bermudagrass. Agricultural Water Management, 87, 328-336. doi: 10.1016/j.agwat.2006.08.004

Gloaguen, T. V.; Forti, M. C.; Lucas, Y.; Montes, C. R.; Gonçalves, R. A. B.; Herpin, U.; Melfi, A. J. 2007. Soil solution chemistry of a Brazilian Oxisol irrigated with treated sewage effluent. Agricultural Water Management, 88, 119-131. doi: 10.1016/j.agwat.2006.10.018

Gwenzi, W.; Munondo, R. 2008. Long-term impacts of pasture irrigation with treated sewage effluent on nutrient status of a sandy soil in Zimbabwe. Nutr. Cycl. Agroecosyst., 82, 197-207. doi: 10.1007/s10705-008-91813

Hadas, A.; Sagiv, B.; Haruvy, N. 1999. Agricultural practices, soil fertility management modes and resultant nitrogen leaching rates under semi-arid conditions. Agricultural Water Management, 42, 81-95. doi: 10.1016/S0378-3774(99)00026-8

IBGE - Instituto Brasileiro de Geografia e Estatistica. Monografias Municipais, Nordeste/Pernambuco - Caruaru, 2015. Disponível em: https://biblioteca.ibge.gov.br/visualizacao/per iodicos/2980/momun_ne_pe_caruaru.pdf.

Acesso em: 20 jul. 2019.
INTERÁGUAS - Programa de Desenvolvimento do Setor Água. Agência Nacional de Águas. Elaboração de Proposta do Plano de Ações para instituir uma política de reúso de efluente sanitário tratado no Brasil. Ministério das Cidades e Instituto Interamericano de Cooperação para a Agricultura - IICA. 2017. Disponível em: http://interaguas.ana.gov.br/Lists/Licitacoes_ Docs/Attachments/224/Produto\%206_Plano $\% 20 \mathrm{de} \% 20 \mathrm{~A} \% \mathrm{C} 3 \% \mathrm{~A} 7 \% \mathrm{C} 3 \% \mathrm{~B} 5 \mathrm{es} \% 20 \mathrm{e} \% 20$ Pol\%C3\%ADtica\%20de\%20Re\%C3\%BAso. pdf. Acesso em: 18 Jul 2019.

Jeong, H.; Kim, H.; Jang, T. 2016 Irrigation water quality standards for indirect wastewater reuse in agriculture: a contribution toward sustainable wastewater reuse in South Korea. Water, 8, 169-186. doi: 10.3390/w8040169

Kiehl, E. J. Fertilizantes orgânicos. 1985. Editora Agronômica Ceres. Piracicaba, São Paulo.

Kiziloglu, F. M.; Turan, M.; Sahin, U.; Kuslu, Y.; Dursun, A. 2008. Effects of untreated and treated wastewater irrigation on some chemical properties of cauliflower (Brassica olerecea L. var. botrytis) and red cabbage (Brassica olerecea L. var. rubra) grown on calcareous soil in Turkey. Agricultural Water Management, 95, 716-734. doi: 10.1016/j.agwat.2008.01.008

Lekfeldt, J. D. S.; Kjaergaard, C. K.; Magid, J. 2017. Long-term effects of organic waste fertilizers on soil structure, tracer transport, and leaching of colloids. Journal of Environmental Quality, 46, 862-870. doi: 10.2134/jeq2016.11.0457

Magesan, G. N.; McLay, C. D. A.; Lal, V. V. 1998. Nitrate leaching from a free-draining volcanic soil irrigated with municipal sewage effluent in New Zealand. Agriculture, Ecosystems and Environment, 70, 181-187. doi: 10.1016/S0167-8809(98)00150-9

Marofi, S.; Shakarami, M.; Rahimi, G.; Ershadfath, F. 2015. Effect of wastewater and compost on leaching nutrients of soil column under basil cultivation. Agricultural Water Management, 158, 266-276. doi: 10.1016/j.agwat.2015.05.007

Massoud, F. I. 1974. Salinity and alkalinity as soil degradation hazards. FAO, Rome, Italy.

Matos, A. T.; Gariglio, H. A. A.; Monaco, P. A. V. L. 2013. Deslocamento miscível de cátions provenientes da vinhaça em colunas de solo. Revista Brasileira de Engenharia Agrícola e Ambiental, 17, 743-749. doi: 10.1590/S141543662013000700008 
Metcalf \& Eddy. 1991. Wastewater engineering. Treatment, disposal, and reuse. McGraw-Hill, New York, USA.

Nguyen, L. A. T.; Ward, A. J.; Lewis, D. 2014. Utilisation of turbidity as an indicator for biochemical and chemical oxygen demand. Journal of Water Process Engineering, 4, 137142. doi: 10.1016/j.jwpe.2014.09.009

Rusan, M. J. M.; Hinnawi, S.; Rousan, L. 2007. Long term effect of wastewater irrigation of forage crops on soil and plant quality parameters. Desalination, 215, 143-152. doi: 10.1016/j.desal.2006.10.032

Sandri, D.; Rosa, R. R. B. 2017. Atributos químicos do solo irrigado com efluente de esgoto tratado, fertirrigação convencional e água de poço. Irriga, 22, 18-33. doi: 10.15809/irriga.2017v22n1p18-33

Semura, K. A.; Riccitelli, M.; Gonçalves, M. C. 2007. Estudo para Implantação de Reúso e Proposição de Parâmetros de Qualidade para Usos Urbanos Não Potáveis a Partir das ETE's da RMSP. Anais do Workshop Uso e Reuso Águas Residuárias e Salinas. Viçosa, MG, Brasil.

Siebe, C. 1998. Nutrients inputs to soils and their uptake by alfafa through long-term irrigation with untreated sewage effluent in Mexico. Soil Use and Management, 14, 119-122. doi: 10.1111/j.1475-2743.1998.tb00628.x

Silva, D. F.; Andrade, C. L. T.; Simeone, M. L. F.; Amaral, T. A.; Castro, L. A.; Moura B. F. 2010. Análise de nitrato e amônio em solo e água. Embrapa Milho e Sorgo, Sete Lagoas, Minas Gerais.

Silva Júnior, W. R.; Sandri, D.; Figueiredo, C. C.; Pereira, R. M. 2019. Chemical attributes of soil irrigated with treated sewage effluent and cultivated with bell pepper. Revista Ambiente \& Água, 14, 1-15. doi: 10.4136/ambiagua. 2341

Silva, F. C. 2009. Manual de análises químicas de solos, plantas e fertilizantes. Embrapa Informação Tecnológica: Embrapa Solos, Rio de Janeiro, Brasil.

Silva, L. P. 2013. Influência do íon acompanhante nos parâmetros de nitrato no solo. Dissertação de Mestrado, Escola Superior de Agricultura Luiz Queiroz. Piracicaba, São Paulo, Brasil. $105 \mathrm{p}$.

SNIRH - Sistema Nacional de Informações sobre Recursos Hídricos. 2019. Disponível em: https://www.ana.gov.br/acesso-asistemas/sistema-nacional-de-informacoessobre-recursos-hidricos-snirh. Acesso em: 24 Jul 2019.

Stutter, M. I. 2015. The composition, leaching, and sorption behavior of some alternative sources of phosphorus for soils. Ambio, 44, 207-216. doi: 10.1007/s13280-014-0615-7

Telles, D. D.; Costa, R. H. P. G. 2007. Reuso da água: Conceitos, teorias e práticas. Editora Blucher, São Paulo.

Urbano, V. R.; Mendonça, T. G.; Bastos, R. G.; Souza, C. F. 2017. Effects of treated wastewater irrigation on soil properties and lettuce yield. Agricultural Water Management, 181, 108-115. doi: 10.1016/j.agwat.2016.12.001

WHO - World Health Organization. 1989. Health guidelines for the use of wastewater in agriculture and aquaculture. Technical Report, series, Geneva, Switzerland. 